\title{
The Design of Android System Based on Instant Communication Software
}

\author{
MU Yuxiang \\ College of Automotive Engineering, Weifang University of Science and Technology, Shouguang, \\ China \\ e-mail: 1253736034@qq.com
}

Keywords-Android, instant messaging,C/S software architecture

\begin{abstract}
In this paper, taking Android system as the operating platform, the design of a high quality can be text, voice and video communication real-time communication software,in connection with the network conditions, can achieve instant communication between all users of Android system. This design can achieve real-time communication between Android platform and Android platform between Android platform and PC.
\end{abstract}

\section{Introduction}

With the rapid development of the popularity of smart mobile phone Android system and mobile communication technology, people require for the ransmission of information communication platform gradually increase, instant communication system with real-time, low cost low, high efficiency advantages in the mobile terminal platform,thus become the mode of network communication are widely use of contemporary young people.

Instant communication system since the appearance of real time communication mode,similar to the network as the foundation,exchange information with other online users, with the characteristics of convenient and fast,got to news feedback real-time demanding users praise.Instant communication software traditional most can be achieved in the PC end,but with the wireless transmission network development and launch a variety of intelligent operation platform,the development of instant messaging system on mobile devices is already 3G/4G business new window.

For quite a long period of time the device with PC mobile terminal instant communication and communication protocol of the lack of uniform standards, this paper in order to solve this problem, the instant communication system is designed and implemented on an Android platform using XMPP protocol.With the rapid development of mobile communication and Internet and the mutual integration, 3G technology enables wireless network and internet to realize high speed connection.Instant communication based on internet protocol, enables applications to communicate in different equipment intelligent mobile phone users, most of the current use of Android operating system, Android system is the mobile phone operating system Linux based on open source software, XMPP is the XML open source instant messaging protocol based on Android platform and XMPP protocol, so the development of instant messaging system based on has application prospect very good.

\section{communication system overview}

The instant communication system using C/S architecture, the use of open-source XMPP as instant messaging protocol, the client uses the TCP protocol to connect to the server, by building the open source Open fire server as the instant communication platform, achieve real-time communication between the client and the client. 


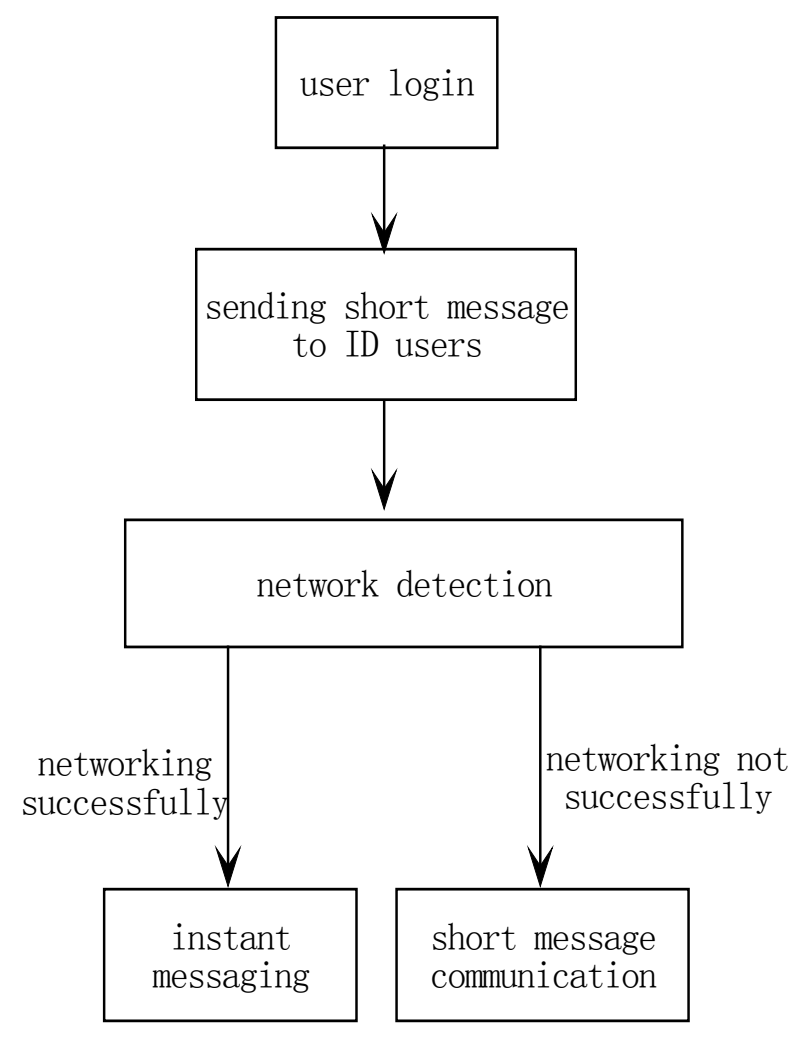

Figure 1. general block diagram communication system

In the design of system communication,instant communication, communication both sides need to be in a connected state radio network,so the system communication, first of all to detect the wireless network connection, through the wireless communication module calls the Android platform, the detection and identification of the current wireless network is connected, if the connection can communicate according to the sender ID, if there is no wireless network connection is not successful, can communicate through the way of short message. The general frame of communication system as shown in figure 1 .

In the process of communication,communication both sides need to be in the same LAN from the group, so the system must first detect the LAN connection, through the wireless communication module calls the Android platform, the detection and identification of the local area network is connected, if the connection is shown to LAN SSID name, and can carry out instant communication mode. When detecting the LAN is not availableor the communication between the ad hoc LAN is not at the same time, the communication system cannot directly an instant message, in order to continue to realize the interactive user information of this case, real-time system will automatically jump to the short message communication mode phone mode, and message communication, normal, implementation communication function in this case.

\section{The design of the communication system}

\section{A. introduction to Android}

Android is Google's announcement of the open source mobile operating system. From an architectural point of view, it was developed specifically for mobile terminals by the Linux kernel, middleware, user interface and application software program composition, it is the first fully open source free and the operating system. Android platform is not only an operating system, but also an nature architecture of open source. Android platform focused on the achievement of all the open source community in one part and for the mobile device of its own do a lot of improvement and optimization. Compared with other mobile operating system, Android has four unparalleled advantages. 
- Platform openness. Android system is a fully open source mobile platform, the most important is that the system itself is free, greatly reducing development costs manufacturers, operators can freely develop new applications, in addition to a lot of third-party mobile developers are also based on this can be found rapid development opportunities.

- Application equality. The beginning of the birth of the Android platform was designed to be a planning application composition platform, which all applications run on Dalvik virtual machine, which provides a corresponding API interface for mutual communication access between resources.

- Application of liquidity. There are no boundaries between Android applications, developers can be linked to the development of a variety of services, such as text messaging, GPS, network services can be connected together.

- Develop quickness. Android offers a lot of that see-use libraries and tools for developers, developers can use these libraries and tools to quickly develop their own applications. Eg .Android platform integrates Google Maps, Google Voice, etc., developers only need to create related applications by calling several lines of code.

The architecture of the Android platform is divided into five parts:the application, application framework, development libraries, the run time environment and the Linux kernel, as shown in figure 2.

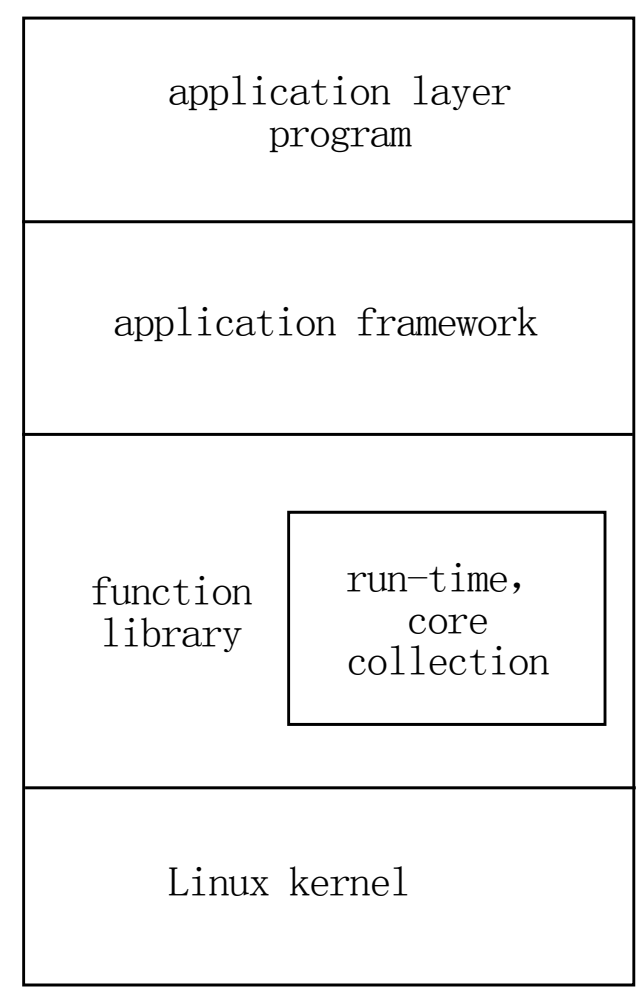

Figure 2. paracelsian paracelsus for Android

The core service system of Android relies on the Linux2.6 kernel, such as security, memory management, process management, network protocol stack and driving model, at the same time, the Linux kernel also as an abstraction layer between the hardware and software of stack. In the preparation of application, can access the Android provides the API box,call its $\mathrm{C} / \mathrm{C}++$ development library and core application package.

\section{B. The functional architecture of instant communication system}

According to the division of the function module of the system, using the C/S system architecture,communication model and the use of P2P in the speech communication. Due to the differences between the functional modules of the system, through the interaction between the Android client and Open fire server, mainly depends on the function of XMPP protocol,instant communication effect is good. As for the requirements of higher real-time audio communication, the real-time point-to-point transmission is dependent on UDP/RTP. 


\section{Hierarchical structure of design client}

This system adopts hierarchical framework of MVC Client software,will the entire client into a display layer, business logic layer and model layer.The logic of the hierarchical structure of the system is divided into display client layer, logic control layer and model layer. The model layer consists of Model data model layer and data access layer for DB. The logic control layer is divided into Util layer, Activity layer, Adapter layer. Among them,the Util layer is the upper layer provides a service interface, and provides the call interface for the Activity layer, to provide services for the main logic module function. The Activity layer is the main logic processing layer system, the function of each module of the logic processing. The Adapter layer is the system used to update the interface level. Display layer is mainly view layer, defined by the Android layout XML file, used to define and describe the user interface. Other Android resource file, related menu items and the resource string also belongs to the display layer.

\section{Detailed design of the client}

The client of the detailed design of the system is the key of the whole system design. Mainly including log in module, voice communication module, file transfer module, chat module part.

The log in module mainly includes the new user registration and the old user log in, the user enters to register a new user interface, fill out the form after you submit, first in a client-side initialization and fill in the form according to the settings of a Registration object, set its type to IQ. Type.SET, and then set the two packet filter filter, respectively, according to Regisition data packets the ID and IQ types of data packet filtering. If the IQ data collected by the package Collector is not empty, and the type is IQ.Type.RESULT, that is registered successfully, you can log on.

Voice communication module mainly realizes the transmission of audio files, users to record an audio, and then sent to the target user, the target users receive audio, click the message box will be on the audio message file playback. The module design, design of recording and sending the two button in the user interface to the ID, hit the record button, initialize the voice interface, and began to collect, the audio release, completed the sound recording, and audio file when the length of the judgment, for more than $2 \mathrm{~S}$ of the audio documents sent to the execution, less than $2 \mathrm{~S}$ file after confirmation sent.

A file transfer module has been used to in the voice message communication module, using the function alone is the main transmission complete text, image file on the mobile phone file, use is the first select the file to obtain a specific file path, and then the path to the file as the parameter, the Smack library function call for file transfer.

Chat module is a communication system to use the highest frequency function. Module for the client to send and receive information, divided into message sending and receiving the two part, mainly by the chat Activity. Java processing mainly logic. Treatment of class chat Activity is also responsible for friends among the voice message communication module.

The process of information transmission and real-time communication system as shown in Figure 3 , the system is ready to send a message, first the network detection, if the network is available, then enter the message and time setting module, and uses the UDP transmission protocol transmission,UDP transmission, the first two communication parties UDP connection and initialization of ; at the receiving end, the system defined for receiving information Activity, UDP port number when the program monitoring information spreads, to receive immediate. When the LAN is not available, the system automatically jump to the SMS sending module,information will be sent by the form of SMS. 


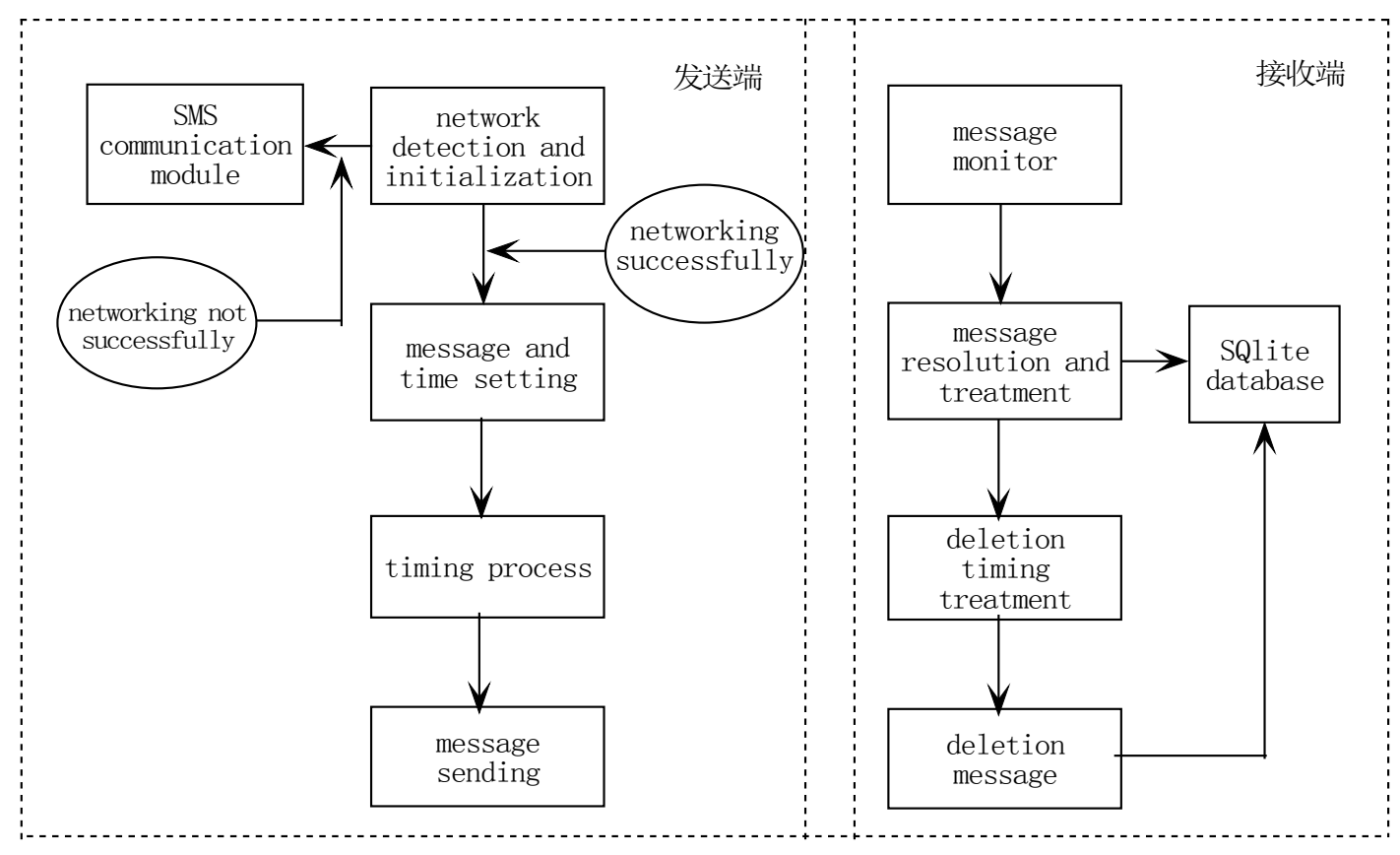

Figure 3. information transmission proce

The client receives the message, mainly is the need to set up a message listener in the client, when processing method by the server to forward messages over will automatically call the monitoring interface. Gets the message object, and then by the handler to handler. Handler according to the type of the message object, different treatment, received ordinary text messages, will chat messages are added to the list of messages, and update the display adapter.

\section{Conclusion}

This paper analyzed the system are hitecture of Android platform and the Android application components and life cycle,deeply studies the methods of kemel-level component software development and applieation software development.

Since 2007, the Android system available, because of its open source,easy to develop, in recent years has been widely applied. Instant communication system of Android platform is realized in communication between LAN users from groups based on design, and develop intelligent timeefficient, and the system can realize the short message communication without network under selective, the instant communication system to the mobile phone short message and mobile phone mobile Internet is the perfect combination of together, the user through the mobile terminal equipment, can conveniently carry on the real-time information exchange and others.

\section{References}

[1] Huang Weimin . Design of Android Instant Messaging Syatem Based on XMPP Agreement[J]. Electronic Design Engineering, 2011.4(8):57-59.

[2] Jiang Shaolin,Wang Jinshuang,Zhang Tao,Chen Rong.A Summary on Android Security[J]. Computer Applications and Software,2012.10.

[3] CHEN Jing,CHEN Ping-hua,LI Wenliang. Analysis of Android Kernel[J]. Modern Computer,2009.11.

[4] Fang Yu,Huang Liang,Chen Shiping. Operation Mode and Development Prospects of Agricultural Information Service Platform Based on Android System[J]. Modern Agricultural Science and Technology.2014.19. 
[5] Yao Yumin,Liu Weiguo. Study of Android's Architecture and its Application Development[J]. Computer Systems \& Applications, 2011(11):110-112.

[6] Wang Nan,Song Fei,Zhou Huachun. An Instant Communication Scheme Based on Android Platform[J]. Computer Application and Software, 2013.4 : 108-109.

[7] Ni Jian,Xu Sheng. Audio and Video Real-time Communication Design and Implementation Based on Android [J]. Journal of Civil Aviation Flight University of China,2013.5 : 48-51.

[8] DONG Tao. The Design and Implementation of Mobile Campus Client Based on Android. A Dissertation Submitted to Xidian University for the Degree of Master,2013.04

[9] FANG Jing. The Design and Implementation of Mobile Learning Platform Based on The Android System. A Dissertation Submitted to Xidian University for the Degree of Master,2014.05

[10] Xiong Gang. The Design and Realization of Smart Phone based on Android. A Dissertation Submitted to Xidian University for the Degree of Master,2010.05

[11] Wu Xiangxiang. Research and Application of software development approaches based on Android platform. A Dissertation Submitted to Beijing University of Posts and Telecommunications for the Degree of Master.2011.01 